\title{
Improved Cryptanalysis of Reduced RIPEMD-160
}

\author{
Florian Mendel ${ }^{1}$, Thomas Peyrin ${ }^{2}$, Martin Schläffer ${ }^{1}$, \\ Lei $\mathrm{Wang}^{2}$, and Shuang $\mathrm{Wu}^{2}$ \\ 1 IAIK, Graz University of Technology, Austria \\ \{florian.mendel, martin.schlaeffer\}@iaik. tugraz.at \\ ${ }^{2}$ Nanyang Technological University, Singapore \\ thomas.peyrin@gmail.com, \\ \{wang. lei, wushuang\}@ntu.edu.sg
}

\begin{abstract}
In this article, we propose an improved cryptanalysis of the double-branch hash function standard RIPEMD-160. Using a carefully designed non-linear path search tool, we study the potential differential paths that can be constructed from a difference in a single message word and show that some of these message words can lead to very good differential path candidates. Leveraging the recent freedom degree utilization technique from Landelle and Peyrin to merge two branch instances, we eventually manage to obtain a semi-free-start collision attack for 42 steps of the RIPEMD-160 compression function, while the previously best know result reached 36 steps. In addition, we also describe a 36-step semi-freestart collision attack which starts from the first step.
\end{abstract}

Keywords: RIPEMD-160, semi-free-start collision, compression function, hash function.

\section{Introduction}

Due to their widespread use in many applications and protocols, hash functions are among the most important primitives in cryptography. A hash function $H$ is a function that takes an arbitrarily long message $M$ as input and outputs a fixed-length hash value of size $n$ bits. Cryptographic hash functions have the extra requirement that some security properties, such as collision resistance and (second)-preimage resistance, must be fulfilled. More precisely, it should be impossible for an adversary to find a collision (two distinct messages that lead to the same hash value) in less than $2^{n / 2}$ hash computations, or a (second)preimage (a message hashing to a given challenge) in less than $2^{n}$ hash computations. Most standardized hash functions are based upon the Merkle-Damgård paradigm 13.3 and iterate a compression function $h$ with fixed input size to handle arbitrarily long messages. The compression function itself should ensure equivalent security properties in order for the hash function to inherit from them.

The cryptographic community have seen very impressive advances in hash functions cryptanalysis in the recent years [20 18 19 17], with weaknesses or even 
sometimes collisions exhibited for many standards such as MD4, MD5, SHA-0 and SHA-1. These functions have in common their design strategy, based on the utilization of additions, rotations, xors and boolean functions in an unbalanced Feistel network. In order to diversify the panel of standardized hash functions and make a backup plan available in case the last survivors of this MD-SHA family gets broken as well, NIST organized a 4-year SHA-3 competition which led to the selection of Keccak [1 as new standardized primitive. The move of the industry towards SHA-3 will take a lot of time and even broken functions such as MD5 or SHA-1 remain widely used. Among the MD-SHA family, only SHA-2 and RIPEMD-160 compression functions are still unbroken, although practical collisions on the SHA-2 compression function have been improved from 24 to 38 steps recently 1112 . The compression function used in RIPEMD-128 was recently shown not to be collision resistant $[8]$.

RIPEMD can be considered as a subfamily of the MD-SHA-family as its first representative, RIPEMD-0 [2], basically consists in two MD4-like 15] functions computed in parallel (but with different constant additions for the two branches), with 48 steps in total. Even though RIPEMD-0 was recommended by the European RACE Integrity Primitives Evaluation (RIPE) consortium, its security was put into question with the early work from Dobbertin [5] and the practical collision attack from Wang et al. 17. Meanwhile, in 1996, Dobbertin, Bosselaers and Preneel [6] proposed two strengthened versions of the original RIPEMD-0, called RIPEMD-128 and RIPEMD-160, with 128/160-bit output and 64/80 steps respectively. RIPEMD-0 main flaw was that its two computation branches were too much similar and this issue was patched in RIPEMD-128 and RIPEMD-160 by using not only different constants, but also different rotation values, boolean functions and message insertion schedules in the two branches. This two-branch structure in RIPEMD family is a good method to reduce the ability of the attacker to properly use the available freedom degrees and to find good differential paths for the entire scheme. RIPEMD-160 is a worldwide ISO/IEC standards [7] that is yet unbroken and is present in many implementations of security protocols.

As of today, the best results on RIPEMD-160 are a very costly 31-step preimage attack [14, a practical 36-step semi-free-start collision attack [10] on the compression function (not starting from the first step), and a distinguisher on up to 51 steps of the compression function with a very high complexity [16].

Our Contributions. In this article, we improve the best know results on RIPEMD-160, proposing a semi-free-start collision attack on 42 steps of its compression function and a semi-free-start collision attack on 36 steps starting from the first step. Our differential paths were crafted thanks to a very efficient nonlinear path search tool (Section 3 ) and by inserting a difference only in a single message word, in a hope for a sparse difference trail. We then explain in Section 4 why we believe the $8^{\text {th }}$ message input word $\left(M_{7}\right)$ is the best candidate for that matter. Once the differential paths settled, we leverage in Section 5 the freedom degree utilization technique introduced by Landelle and Peyrin [8] for RIPEMD-128 that merges two branch instances together in order to obtain a 
semi-free-start collision. It is to be noted that the step function of RIPEMD-160 makes it much more difficult to find a collision attack compared to RIPEMD-128. This is mainly due to the fact that the diffusion is better, but also because even though differences might be absorbed in the boolean function, they will propagate anyway at least once through a free term (which was not the case in RIPEMD-128). We give a description of RIPEMD-160 in Section 2 and summarize our results in Section 6 .

\section{Description of RIPEMD-160}

RIPEMD-160 [6] is a 160-bit hash function that uses the Merkle-Damgård construction as domain extension algorithm: the hash function is built by iterating a 160-bit compression function $h$ that takes as input a 512-bit message block $m_{i}$ and a 160-bit chaining variable $c v_{i}$ :

$$
c v_{i+1}=h\left(c v_{i}, m_{i}\right)
$$

where the message $m$ to hash is padded beforehand to a multiple of 512 bit:1 and the first chaining variable is set to a predetermined initial value $c v_{0}=I V$.

We refer to [6] for a complete description of RIPEMD-160. In the rest of this article, we denote by $[Z]_{i}$ the $i$-th bit of a word $Z$, starting the counting from 0 . $\boxplus$ and $\boxminus$ represent the modular addition and subtraction on 32 bits, and $\oplus, \vee$, $\wedge$, the bitwise "exclusive or", the bitwise "or", and the bitwise "and" function respectively.

\subsection{RIPEMD-160 Compression Function}

The RIPEMD-160 compression function is a wider version of RIPEMD-128, which is in turn based on MD4, but with the particularity that it uses two parallel instances of it. We differentiate these two computation branches by left and right branch and we denote by $X_{i}$ (resp. $Y_{i}$ ) the 32-bit word of left branch (resp. right branch) that will be updated during step $i$ of the compression function. The compression function process is composed of 80 steps divided into 5 rounds of 16 steps each in both branches.

Initialization. The 160-bit input chaining variable $c v_{i}$ is divided into 5 words $h_{i}$ of 32 bits each, that will be used to initialize the left and right branch 160-bit internal state:

$$
\begin{array}{lllll}
X_{-4}=\left(h_{0}\right) \gg 10 & X_{-3}=\left(h_{4}\right) \gg 10 & X_{-2}=\left(h_{3}\right) \gg 10 & X_{-1}=h_{2} & X_{0}=h_{1} \\
Y_{-4}=\left(h_{0}\right) \gg 10 & Y_{-3}=\left(h_{4}\right) \gg 10 & Y_{-2}=\left(h_{3}\right) \gg 10 & Y_{-1}=h_{2} & Y_{0}=h_{1} .
\end{array}
$$

\footnotetext{
${ }^{1}$ The padding is the same as for MD4: a " 1 " is first appended to the message, then $x$ "0" bits (with $x=512-(|m|+1+64(\bmod 512))$ ) are added, and finally the message length $|m|$ coded on 64 bits is appended as well.
} 
The Message Expansion. The 512-bit input message block is divided into 16 words $M_{i}$ of 32 bits each. Each word $M_{i}$ will be used once in every round in a permuted order (similarly to MD4 and RIPEMD-128) and for both branches. We denote by $W_{i}^{l}$ (resp. $W_{i}^{r}$ ) the 32-bit expanded message word that will be used to update the left branch (resp. right branch) during step $i$. We have for $0 \leq j \leq 4$ and $0 \leq k \leq 15$ :

$$
W_{j \cdot 16+k}^{l}=M_{\pi_{j}^{l}(k)} \quad \text { and } \quad W_{j \cdot 16+k}^{r}=M_{\pi_{j}^{r}(k)}
$$

where $\pi_{j}^{l}$ and $\pi_{j}^{r}$ are permutations.

The Step Function. At every step $i$, the registers $X_{i+1}$ and $Y_{i+1}$ are updated with functions $f_{j}^{l}$ and $f_{j}^{r}$ that depend on the round $j$ in which $i$ belongs:

$$
\begin{aligned}
& X_{i+1}=\left(X_{i-3}\right)^{\ll<10} \boxplus\left(\left(X_{i-4}\right)^{\ll \ll 10} \boxplus \Phi_{j}^{l}\left(X_{i}, X_{i-1},\left(X_{i-2}\right)^{\ll \ll 10}\right) \boxplus W_{i}^{l} \boxplus K_{j}^{l}\right)^{\ll \ll s_{i}^{l}}, \\
& Y_{i+1}=\left(Y_{i-3}\right)^{\ll \ll 10} \boxplus\left(\left(Y_{i-4}\right)^{\ll<10} \boxplus \Phi_{j}^{r}\left(Y_{i}, Y_{i-1},\left(Y_{i-2}\right)^{\ll<10}\right) \boxplus W_{i}^{r} \boxplus K_{j}^{r}\right)^{\ll<s_{i}^{r},}
\end{aligned}
$$

where $K_{j}^{l}, K_{j}^{r}$ are 32 -bit constants defined for every round $j$ and every branch, $s_{i}^{l}, s_{i}^{r}$ are rotation constants defined for every step $i$ and every branch, $\Phi_{j}^{l}, \Phi_{j}^{r}$ are 32-bit boolean functions defined for every round $j$ and every branch.

The Finalization. A finalization and a feed-forward is applied when all 80 steps have been computed in both branches. The four 32-bit words $h_{i}^{\prime}$ composing the output chaining variable are finally obtained by:

$$
\begin{array}{cccc}
h_{0}^{\prime}= & h_{1} \boxplus X_{79} \boxplus\left(Y_{78}\right)^{\ll<10} & h_{1}^{\prime}= & h_{2} \boxplus\left(X_{78}\right)^{\ll<10} \boxplus\left(Y_{77}\right)^{\ll<10} \\
h_{2}^{\prime}= & h_{3} \boxplus\left(X_{77}\right)^{\ll<10} \boxplus\left(Y_{76}\right)^{\ll<10} & h_{3}^{\prime}= & h_{4} \boxplus\left(X_{76}\right)^{\ll<10} \boxplus Y_{80} \\
h_{4}^{\prime}= & h_{0} \boxplus X_{80} \boxplus Y_{79} & &
\end{array}
$$

\section{Non-linear Path Search}

To find a non-linear differential path in RIPEMD-160, we use the techniques developed by Mendel et al. [11/2]. This automated search algorithm can be used to find both, differential characteristics and conforming message pairs (note that we will use it only for the differential characteristics part in this article). We briefly describe the tool in Section 3.1, and the new improvements and specific configuration for RIPEMD-160 and our attack in Section 3.2.

\subsection{Automated Search for Differential Characteristics}

The basic idea of the search algorithm is to pick and guess previously unrestricted bits. After each guess, the information due to these restrictions is propagated to other bits. If an inconsistency occurs, the algorithm backtracks to an earlier state of the search and tries to correct it. Similar to [112, we denote these three parts of the search by decision (guessing), deduction (propagation), and backtracking (correction). Then, the search algorithm proceeds as follows: 


\section{Decision (Guessing)}

1. Pick randomly (or according to some heuristic) an unrestricted decision bit.

2. Impose new constraints on this decision bit.

\section{Deduction (Propagation)}

3. Propagate the new information to other variables and equations as described in 11,12.

4. If an inconsistency is detected start backtracking, else continue with step 1 .

\section{Backtracking (Correction)}

5. Try a different choice for the decision bit.

6. If all choices result in an inconsistency, mark the bit as critical.

7. Jump back until the critical bit can be resolved.

8. Continue with step 1.

During the search, we mainly use generalized conditions 4] to store, restrict and propagate information. The decision of choosing which bits to guess depends strongly on the specific attack, hash function and preferred resulting path. E.g. if some parts of the non-linear path should be especially sparse, we guess the corresponding state words first.

Similar to [1112, new restrictions are propagated using brute-force propagation within bitslices for each Boolean function and modular addition. In the backtracking, we remember a small set of critical bits and repeatedly check if all of them can be resolved. This way, we leave dead search branches faster. Additionally, we restart the search after a certain number of inconsistencies occur.

The main difficulty in finding a long differential characteristic lies in the finetuning of the search algorithm. There are a lot of variations possible which can decide whether the search eventually succeeds or fails. We describe the specific improvements for RIPEMD-160 in the next section.

\subsection{Improvements for RIPEMD-160}

To efficiently find non-linear differential paths and message pairs for a larger number of steps than in previous attacks [10, we had to improve the search in several ways. Especially finding a non-linear path for the XOR-round of RIPEMD-160 was quite challenging.

In order to improve the propagation of information, we have combined the bitslices of the two modular additions in each step of RIPEMD-160 into a single bitslice. The two carries of the first and second modular addition are computed and stored together within a generalized 3-bit condition, which is defined similarly as the 2.5-bit condition of [9]. Without this combination, many contradictions would be detected very late during the search, and therefore reduce the overall performance.

To find sparser paths at the beginning or end of the non-linear path, we first propagate the single bit condition in the message word backward and forward more or less linearly and by hand. Then, the automatic search tool is used to connect the paths. Note that due to the additional modular addition in RIPEMD-160, 
we can stay sparse longer in forward direction than in backward direction. This can be observed when looking at our resulting differential paths in Appendix A

Once we have found a candidate for a differential path, we immediately continue the search for partial confirming message pairs, similar as in [1112]. We first pick decision bits '-' which are constraint by linear two-bit conditions of the form $\left(X_{i, j}=X_{k, l}\right.$ or $\left.X_{i, j} \neq X_{k, l}\right)$. This ensures that those bits which influence a lot of other bits are guessed first. This way, inconsistent characteristics are found faster and can also be corrected by the backtracking step of the path search.

\section{Differential Paths}

The previous semi-free-start collision attacks on RIPEMD compression functions usually start by spending the available freedom degrees in the first steps in each branch, and then continue the computation in the forward direction, verifying the rest of the differential path in each branch probabilistically. With this attack strategy, the non-linear part of the differential paths for both branches should be located in the early steps. Indeed, the non-linear parts are usually the most costly part and therefore should be handle in priority by the attack with the available freedom degrees.

Since the compression functions belonging to the RIPEMD family use a twobranch parallel structure sharing the same initial chaining value, the left and right branches can be regarded as somehow connected in the first steps. With this observation, in [8] Landelle and Peyrin proposed a new method to find semifree-start collisions for RIPEMD-128. Their method allows the attacker to use the message freedom degrees not necessarily in the early steps of each branch, and therefore relax a bit the constraint that the most costly parts (the non-linear chunks) must be located in the early steps as well. Consequently, the space of possible differential paths is increased and likely to contain better candidates since the probabilistic part in each branch is reduced.

Figure 1 shows the difference between the previous and the new strategies. The attack process proposed in [8] is made of three steps. Firstly, the attacker independently choose the internal states in both branches and start fixing some message words in order to handle the two non-linear parts. Then, he uses some of the remaining message words available to merge the two branches to the same chaining variable by computing backward from the middle. Finally, the rest of the differential path in both branches is verified probabilistically by computing forward from the middle.

\subsection{On the Choice of the Message Word}

As in [8], in order to find a sparse differential path for a semi-free-start collision attack with the biggest number of steps, we chose to insert differences in only a single message word. Then, for all the 16 message words, we have analyzed how many steps can be potentially attacked. The results are summarized in Table 1 Note that the details of the attacks are not considered at this stage and the final 

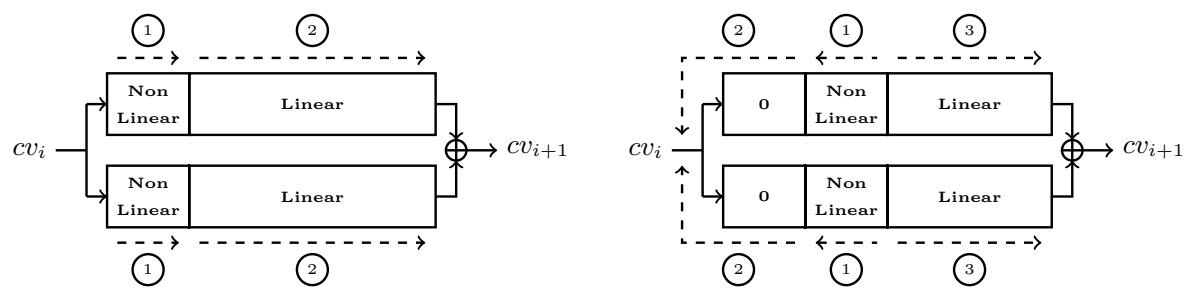

Fig. 1. The previous (left-hand side) and new (right-hand side) approach for collision search on double-branch compression functions introduced in [8]

complexity will highly depend on the merging process and the quality of the differential paths that can be found (both linear and non-linear parts). Yet, by guessing for each message insertion where would be the best location for the two non-linear parts, this preliminary analysis gives a rough estimation of how many steps can be reached potentially. The overall attack being quite complex and time consuming to settle, this will help us to focus directly on good candidates.

We found that message words $M_{7}$ and $M_{14}$ both seem to be rather good choices when trying to verify the following criteria:

1. the non-linear part in both branches should be short, in order to consume less freedom degrees.

2. the early steps of the two non-linear parts should be rather close to each other, which will help the merging.

3. the late steps of the non-linear parts should be as sparse as possible, since after the merging comes the probabilistic phase and ensuring a sparse incoming difference mask would guarantee a rather high differential probability when computing forward.

4. some message word difference injections allow the differences injected in very late steps in the two branches to cancel each other through the final feedforward operation. If this trick is applicable, one can usually get 4 to 6 extra steps for the collision attack with a relatively low cost.

Once $M_{7}$ and $M_{14}$ identified as good candidates, we tried to design the entire differential path and establish the merging phase. During our search for the linear part of the differential path, we found it much harder to find good ones for RIPEMD-160 compared to RIPEMD-128. The reason is that the diffusion of the step function of RIPEMD-160 is much better than RIPEMD-128 as it prevents from fully and directly absorbing all the differences. For example, a step in an IF round in RIPEMD-128 can be fully controlled by the attacker such that no difference diffusion occurs. However, in RIPEMD-160, one extra free term appears in the addition of the step function formula and this forces at least a diffusion of a factor two (that cannot be absorbed by the IF function). As a consequence, we were not able to find differential paths as sparse as in [8] and the number of attacked steps is also much lower. 
Table 1. Rough estimation of the number of attackable steps for various choices of message words differences injection (in parenthesis are given the steps window)

\begin{tabular}{|c|c|c|c|c|}
\hline Message Word & $M_{0}$ & $M_{1}$ & $M_{2}$ & $M_{3}$ \\
\hline Attackable Steps & $51(26-76)$ & $46(2-47)$ & $52(6-57)$ & $48(4-51)$ \\
\hline
\end{tabular}

\begin{tabular}{|c|c|c|c|c|}
\hline Message Word & $M_{4}$ & $M_{5}$ & $M_{6}$ & $M_{7}$ \\
\hline Attackable Steps & $42(8-49)$ & $50(6-55)$ & $39(10-48)$ & $56(8-63)$ \\
\hline
\end{tabular}

\begin{tabular}{|c|c|c|c|c|}
\hline Message Word & $M_{8}$ & $M_{9}$ & $M_{10}$ & $M_{11}$ \\
\hline Attackable Steps & $36(12-47)$ & $39(10-48)$ & $37(14-50)$ & $38(12-49)$ \\
\hline
\end{tabular}

\begin{tabular}{|c|c|c|c|c|}
\hline Message Word & $M_{12}$ & $M_{13}$ & $M_{14}$ & $M_{15}$ \\
\hline Attackable Steps & $38(16-53)$ & $34(41-74)$ & $58(2-59)$ & $43(11-53)$ \\
\hline
\end{tabular}

\subsection{Difficulty of Calculating the Probability}

Another important difference between RIPEMD-128 and RIPEMD-160 is the step differential probability calculation. While it is easy to calculate the differential probability for each step of a given differential path of RIPEMD-128, it is not the case for RIPEMD-160. The reason is that the step function in RIPEMD-160 is no longer a S-function (a function for which the $i$-th output bit depends only on the $i$ first lower bits of all input words), and therefore the accurate calculation of the differential probability is very hard. Yet, one can write the step function as two S-functions by introducing a temporary state that we denote $Q_{i}$. We use the step function of the left branch as an example:

$$
\begin{aligned}
Q_{i} & =\left(X_{i-4}\right)^{\ll<10} \boxplus \Phi_{j}^{l}\left(X_{i}, X_{i-1},\left(X_{i-2}\right)^{\ll<10}\right) \boxplus W_{i}^{l} \boxplus K_{j}^{l}, \\
X_{i+1} & =\left(X_{i-3}\right)^{\ll<10} \boxplus Q_{i}^{\ll s_{i}^{l}} .
\end{aligned}
$$

Now the probability of the sub-steps can be calculated precisely. One possible way to calculate the probability of the step function is to specify the conditions on $Q_{i}$ and obtain $\operatorname{Pr}\left[X_{i} \rightarrow Q_{i}\right] \cdot \operatorname{Pr}\left[Q_{i} \rightarrow X_{i+1}\right]$ as the step probability.

In fact, this estimation of the probability is not correct. First, there is no freedom degree injected in the step $Q_{i} \rightarrow X_{i+1}$, which means it is not independent from the step of $X_{i} \rightarrow Q_{i}$. Thus their probability can not be calculated as a simple multiplication. Even if this estimation is accurate, it will only represent a lower bound of the real probability, since there could be a lot of possible equivalent characteristics on $Q_{i}$ and only one is taken in account here. We used experiments to estimate the real probability and found that the probabilities obtained using the first method is much lower than the real probability observed when running the attack.

We then tried to come up with another way to calculate the step differential probability. We summed the probabilities of the two sub-steps for all possible characteristics on $Q_{i}$, i.e. we used $\Sigma_{Q_{i}}\left(\operatorname{Pr}\left[X_{i} \rightarrow Q_{i}\right] \cdot \operatorname{Pr}\left[Q_{i} \rightarrow X_{i+1}\right]\right)$ as differential probability for a step. The calculated probability turned out to be much 
higher than the real one. This is explained by the fact that characteristics on $Q_{i}$ in different steps will sometimes introduce conditions on $X_{i}$ and there could be contradictions between some of the conditions. In the calculation, we did not consider the compatibility between the $Q_{i}$ in different steps. It is therefore not surprising that the calculated probability is much higher.

In the following sections, all the probabilities given were obtained by experiments while testing random samples. We leave the problem of theoretically calculating the real step differential probability as an open problem.

\subsection{8-Step Semi-Free-Start Collision Path}

We eventually chose $M_{7}$ as message word for the single difference insertion and the shape of the differential path that we will use can be found in Figure 2 . The non-linear parts are located between steps 16-41 and 19-36 for left the right branch respectively. In steps 58-64, after a linear propagation of the difference injected by $M_{7}$, the differences in the output internal state are suitable to apply the feed-forward trick that allows us to get a collision on the output of the compression function (at the end of step 64). The complete differential path is displayed in Figure 5 in Appendix.

Note that this differential path does not necessarily require to be followed until step 64 to find a collision (thanks to the feed-forward trick). Indeed, by stopping 6 steps before (step 58), the last difference insertions from $M_{7}$ will be removed and no difference will be present in the internal states in both branches (therefore leading directly to a collision, without even using the feed-forward trick). We did a measurement and found that the collision probability for the feed-forward trick (from step 58 to 64 ) is about $2^{-11.3}$. However, our attacks requiring already a lot of operations, we have to remove these extra 6 steps and aim for a 42-step semi-free-start collision attack instead. Yet, one should keep in mind that a rather small improvement with regards to the attack complexity would probably lead to the direct obtaining of a 48-step semi-free-start collision attack by putting back the 6 extra steps. The details of the attack will be given in the next section.

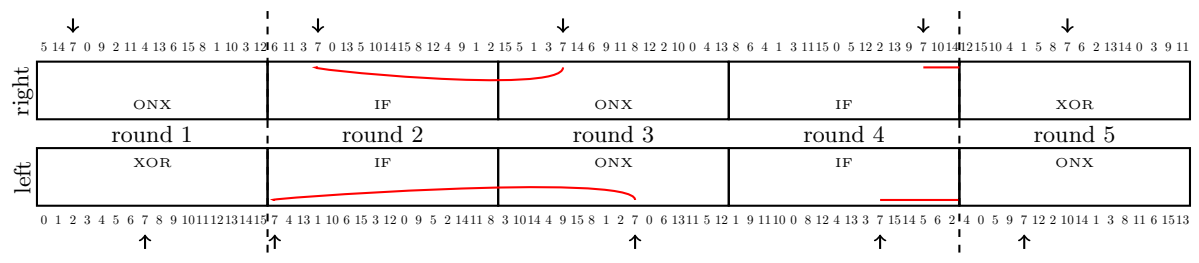

Fig. 2. The shape of our 48-step differential path for the semi-free-start collision attack on the RIPEMD-160 compression function. The numbers represent the message words inserted at each step and the red curves represent the rough amount of differences in the internal state during each step. The arrows show where the bit differences are injected with $M_{7}$. The dashed lines represent the limits of the steps attacked. 


\subsection{6-Step Semi-Free-Start Collision Path from the First Step}

Besides the 48-step path, we also exhibit a semi-free-start collision path starting from the first step, which also use message word $M_{7}$ to introduce differences. Since the boolean function in the first round of the left branch is XOR, it is quite hard to find a non-linear differential path. As a consequence, the path we were able to find turns out to have three bits of differences in $M_{7}$ instead of a single one. The local collisions are located between the first two injections of $M_{7}$ in both branches, thus one can directly derive a 36-step collision path starting from the very first step of RIPEMD-160. Figure 3 shows the shape of this differential path and the detailed path is given in Figure [ in Appendix.

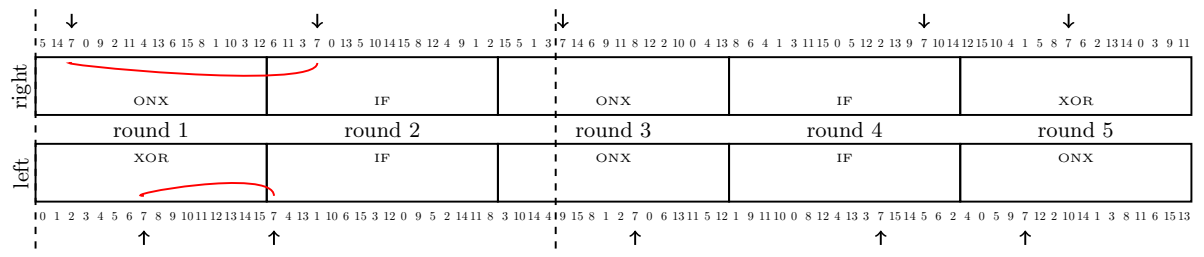

Fig. 3. The shape of our differential path for the 36-step semi-free-start collision attack on the RIPEMD-160 from the first step. The numbers are the message words inserted at each step and the red curves represent the rough amount differences in the internal state during each step. The arrows show where the bit differences are injected with $M_{7}$. The dashed lines represent the limits of the steps attacked.

\section{$5 \quad$ Merging the Two Branches}

Once the differential path is set, we need not only to find conforming pairs for both branches, but also to merge the two branches in order to make sure that they will reach the same chaining variables on their input. Note that for a semifree-start collision, one only needs to ensure that the input chaining variables for both branches are the same and the attacker can actually choose this value freely. In contrary, for a hash collision, the attacker would have to merge both branches to the same chaining variable, fixed to a certain predefined value.

\subsection{Semi-Free-Start Collision}

As explained in previous sections, even though an interesting 48-step differential path has been found (Figure 5), we will only look for a 42-step semi-free-start collision attack on RIPEMD-160, since the feed-forward collision trick would increase the attack complexity beyond the birthday bound. Our algorithm to find a semi-free-start collision is separated in three phases, which we quickly describe here as a high-level view: 
- Phase 1: fix some bits of the message words and the internal states in both branches as preparation for the next phases of the attack. This will allow us to fulfill in advance some conditions of the differential path.

- Phase 2: fix the internal state variables $X_{26}, X_{27}, X_{28}, X_{29}, X_{30}$ of the left branch and $Y_{21}, Y_{22}, Y_{23}, Y_{24}, Y_{25}$ of the right branch. Then, iteratively fix message words $M_{11}, M_{15}, M_{8}, M_{3}, M_{12}, M_{14}, M_{10}, M_{2}, M_{5}, M_{9}, M_{0}$ and $M_{6}$ in this particular order, so as to fulfill the conditions located inside or close to the non-linear parts. Once these internal state variables or message words are successfully fixed, we call this candidate at the end of phase 2 a starting point for the merging.

- Phase 3: use the remaining free message words $M_{1}, M_{4}, M_{7}$ and $M_{13}$ to merge the internal states of both branches to the same input chaining value. Since every value is fixed at this point, check if the rest of the differential path is fulfilled as well (the uncontrolled part).

Phase 1: Preparation. Before finding a starting point for the merging, we can prepare the differential path by introducing certain conditions on the internal states in both branches in order to increase the probability of the uncontrolled part of the differential path.

The condition that we will force is that bits 16 to 25 of $X_{35}$ must be equal to 0 n00n00000. The effect of this condition is that when a starting point will be generated, we will be able to directly deduce the 8 lowest bits of $X_{37}$ only by fixing bits 16 to 25 of $M_{9}$. In order to explain this, note that calculating $X_{37}$ during the step function in the forward direction gives:

$$
X_{37}=X_{33}^{\lll 10} \boxplus\left(X_{32}^{\lll 10} \boxplus \operatorname{ONZ}\left(X_{36}, X_{35}, X_{34}\right) \boxplus M_{9} \boxplus K_{36}^{l}\right) \lll 14
$$

Since $\operatorname{ONZ}\left(X_{36}, X_{35}, X_{34}\right)=\left(X_{36} \vee \overline{X_{35}}\right) \oplus X_{34}$, bits 16 to 25 of $X_{36}$ will have no influence on the output of the boolean function ONZ if the corresponding $X_{35}$ bits are set to zero (in a starting point, $X_{32}, X_{33}, X_{34}$ and $X_{35}$ are already fully known). Then, we can choose $M_{9}$ such that bit 16 of $X_{32}^{\lll 10} \boxplus\left(\left(\left(X_{36} \vee \overline{X_{35}}\right) \oplus\right.\right.$ $\left.\left.X_{34}\right) \& 3 \mathrm{ff}\right) \boxplus\left(M_{9} \& 3 \mathrm{ff}\right) \boxplus K_{36}$ equals zero, which will stop the carry coming from the lower bits. As a result, the 8 lowest bits of $X_{37}$ will not depend on $X_{36}$ anymore (and thus neither on $M_{4}$ when computing forward, since $X_{36}$ directly depends on $M_{4}$ ).

One example of our generated starting points is shown in Figure 4, in which we applied our preparation trick. Before generating this starting point, we forced the additional conditions on $X_{35}$, and once the starting point found, fixing bits 16 to 25 of $M_{9}$ to 01101000010 will make sure that the last 8 bits of $X_{37}$ will be equal to 11111010 . Note that the 26-th bit of $M_{9}$ and 9 -th bit of $X_{37}$ are deduced from the known conditions.

Applying this trick is interesting for the attacker because the uncontrolled probability (steps 35-58) of the left branch is increased.

Phase 2: Finding a Starting Point. Given the differential path from Figure 5 we can use the freedom degrees available in both left and right branches internal 


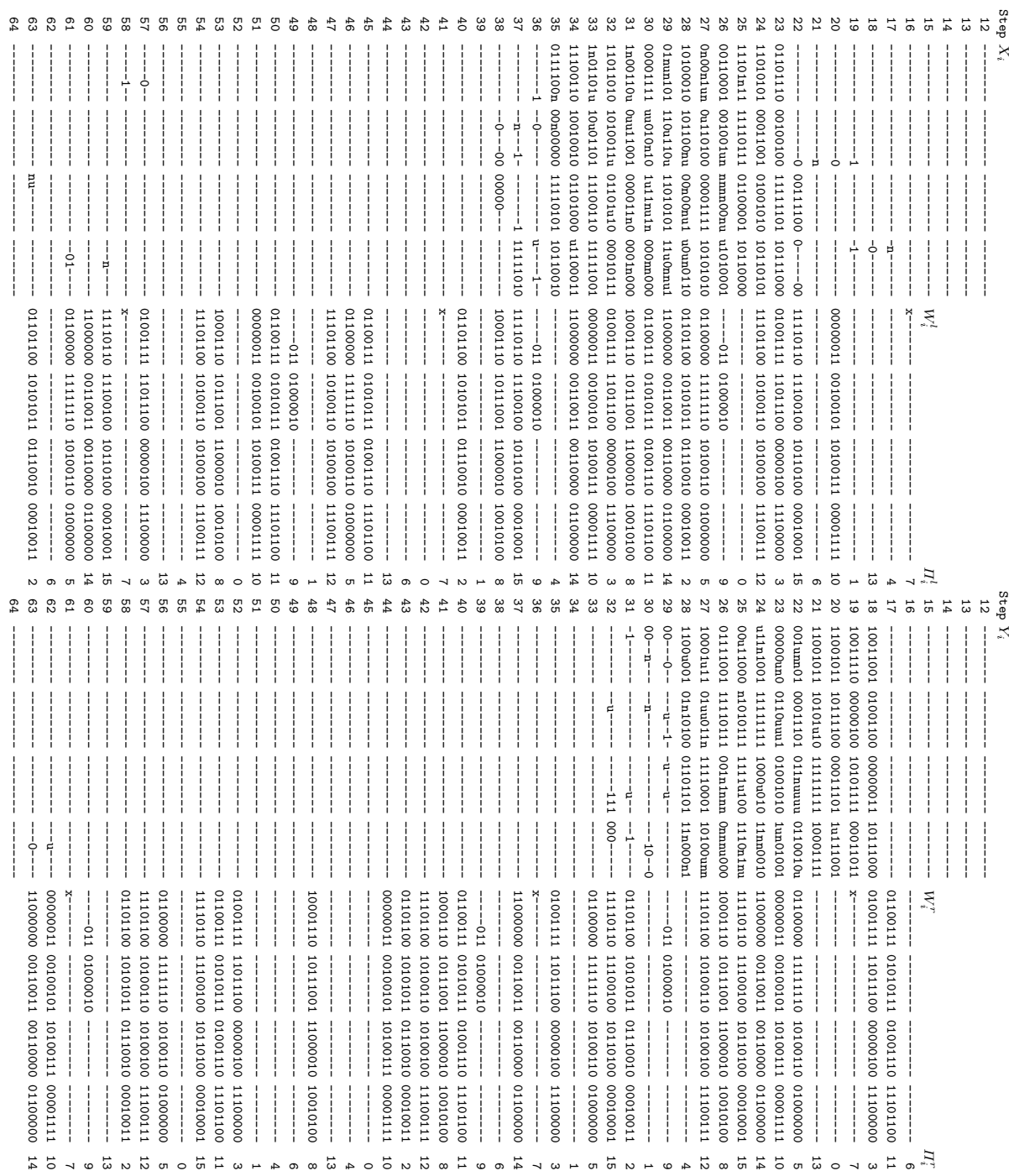

Fig. 4. Starting point for the 48-step differential path, on which the preparation trick was applied and the last 8 bits of $X_{37}$ are fixed in advance by choosing several bits of $M_{9}$. At this point the remaining free message words are $M_{9}, M_{0}, M_{6}, M_{13}, M_{1}, M_{7}$ and $M_{4}$, which will be used during the merging phase.

states (320 bits) and in the message words (512 bits) to fulfill as much differential conditions as possible. To make the attacker easier, we chose to fix first the five consecutive internal states words that contain the most differential conditions $\left(X_{26}, X_{27}, X_{28}, X_{29}, X_{30}\right.$ in the left branch and $Y_{21}, Y_{22}, Y_{23}, Y_{24}, Y_{25}$ in the right branch). Then, we fix a few message words one by one in the given order and 
continue the computation of the internal states by computing forward and backward in both branches (from $X_{26}, X_{27}, X_{28}, X_{29}, X_{30}$ and $Y_{21}, Y_{22}, Y_{23}, Y_{24}, Y_{25}$ )

Fixing the first 5 chaining values $\left(X_{26}, X_{27}, X_{28}, X_{29}, X_{30}\right.$ of the left branch and $Y_{21}, Y_{22}, Y_{23}, Y_{24}, Y_{25}$ of the right branch) is quite an easy task. Note that the two branches can be fixed independently at this stage. We used algorithms similar to the ones searching for a differential path: we just guess the unrestricted bits - from lower step to higher step, lower bit to higher bit and check if any inconsistency occurs. If both 0 and 1 selection of one bit lead to an inconsistency, we apply the backtracking in the search tree by one level and guess the same bit again. The guessing continues until all bits are fixed. If after a predefined number of backtracking events (chosen according to the performance of the search) no solutions are found, we can restart the whole search in order to avoid being trapped in a bad subspace with no solution at all.

Concerning the fixing of the message words, we used a different approach. Here, our search was applied word by word. Following this message words order$\operatorname{ing} M_{11}, M_{15}, M_{8}, M_{3}, M_{12}, M_{14}, M_{10}, M_{2}, M_{5}, M_{9}, M_{0}$ and $M_{6}$, we guess the free bits, and some internal states values will directly be deduced by computing in both forward and backward directions from the already known internal state values in both branches. Note that the two branches are not independent anymore at this stage (since all message words are added several times in both branches), so it is important to check often for any inconsistency that could be detected. The backtracking and restarting options are also helpful here. We can use an extra trick to get a performance improvement of the search by pre-fixing the value of the word with the biggest number conditions in it (either message word or internal state word), and then deduce the value from all the words involved in this computation.

Our tool can find a starting point in a couple of minutes, with a program not really optimized. We will discuss about the complexity to generate the starting points in the next section.

Phase 3: Merging Both Branches with $M_{1}, M_{4}, M_{7}$ and $M_{13}$. A starting point example is given in Figure 4. Our target is to use the remaining free message words $M_{1}, M_{4}, M_{7}$ and $M_{13}$ to make sure that we have a perfect match on the values of the five initial chaining words of both branches, i.e. $X_{i}=Y_{i}$ for $i \in\{12,13,14,15,16\}$ (the indexes started at 12 because we are not attacking from the first step here). The merging consists of four phases and in order to ease the reading we marked the free message words with colors in each phase. Once their values are fixed, we use black color for them.

- Step 1: Use $M_{13}$ to ensure $X_{16}=Y_{16}$. As one can see, the value of $X_{16}$ is already fixed at this point. Now, observe the two backward step functions of $Y_{17}$ and $Y_{16}$ :

$$
\begin{aligned}
& Y_{17}^{\lll 10}=\left(Y_{22} \boxminus Y_{18}^{\lll 10}\right)^{\ggg 8} \boxminus \operatorname{IFZ}\left(Y_{21}, Y_{20}, Y_{19}^{\lll 10}\right) \boxminus K_{21}^{r} \boxminus M_{13} \\
& Y_{16}^{\lll 10}=\left(Y_{21} \boxminus Y_{17}^{\lll 10}\right)^{\ggg 12} \boxminus \operatorname{IFZ}\left(Y_{20}, Y_{19}, Y_{18}^{\lll 10}\right) \boxminus K_{20}^{r} \boxminus M_{0}
\end{aligned}
$$


Incorporating the equation $X_{16}=Y_{16}$, we can direcly calculate the value of $M_{13}$ from the known ones:

$$
\begin{aligned}
& M_{13}=\quad\left(X_{16}^{\lll 10} \boxplus \operatorname{IFZ}\left(Y_{20}, Y_{19}, Y_{18}^{\lll 10}\right) \boxplus K_{20}^{r} \boxplus M_{0}\right)^{\lll 12} \\
& \boxminus Y_{21} \boxplus\left(Y_{22} \boxminus Y_{18}^{\lll 10}\right) \ggg 8 \boxminus \operatorname{IFZ}\left(Y_{21}, Y_{20}, Y_{19}^{\lll 10}\right) \boxminus K_{21}^{r} \text {. }
\end{aligned}
$$

- Step 2: Similarly, use $M_{1}$ and $M_{7}$ to ensure conditions $X_{15}=Y_{15}$ and $X_{14}=$ $Y_{14}$. Observing the step functions:

$$
\begin{aligned}
& X_{15}^{\lll 10}=\left(X_{20} \boxminus X_{16}^{\lll 10}\right) \ggg 13 \boxminus \operatorname{IFX}\left(X_{19}, X_{18}, X_{17}^{\lll 10}\right) \boxminus K_{19}^{l} \boxminus M_{1} \\
= & Y_{15}^{\lll 10}=\left(Y_{20} \boxminus Y_{16}^{\lll 10}\right) \ggg 7 \boxminus \operatorname{IFZ}\left(Y_{19}, Y_{18}, Y_{17}^{\lll 10}\right) \boxminus K_{19}^{r} \boxminus M_{7} \\
& X_{14}^{\lll 10}=\left(X_{19} \boxminus X_{15}^{\lll 10}\right) \ggg 8 \boxminus \operatorname{IFX}\left(X_{18}, X_{17}, X_{16}^{\lll 10}\right) \boxminus K_{18}^{l} \boxminus M_{13} \\
= & Y_{14}^{\lll 10}=\left(Y_{19} \boxminus Y_{15}^{\lll 10}\right) \ggg 15 \boxminus \operatorname{IFZ}\left(Y_{18}, Y_{17}, Y_{16}^{\lll 10}\right) \boxminus K_{18}^{r} \boxminus M_{3}
\end{aligned}
$$

and introducing notations for the constants, the equations above are simplified to

$$
\begin{aligned}
A \boxminus M_{1} & =B \boxminus M_{7} \\
\left(X_{19} \boxminus\left(A \boxminus M_{1}\right)\right)^{\ggg 8} \boxminus D & =\left(Y_{19} \boxminus\left(B \boxminus M_{7}\right)\right)^{\ggg 15} \boxminus E
\end{aligned}
$$

Let $X=\left(X_{19} \boxminus\left(A \boxminus M_{1}\right)\right) \ggg 8, C_{0}=E \boxminus D$ and $C_{1}=Y_{19} \boxminus X_{19}$. The above equations become one:

$$
X \boxplus C_{0}=\left(C_{1} \boxplus X^{\lll 8}\right) \ggg 15
$$

where $C_{0}$ and $C_{1}$ are constants. The problem of finding the value of $M_{1}$ and $M_{7}$ is equivalent to solving this equation. We find that this equation can be solved with $2^{9}$ computations: we can solve this equation for all $2^{64}$ possible values of $C_{0}$ and $C_{1}$ and store the solutions $\left(M_{1}\right.$ and $\left.M_{7}\right)$ in a big look-up table. Building and storing this table requires $2^{73}$ time and $2^{64}$ memory.

- Step 3: Use $M_{4}$ to ensure $X_{13}=Y_{13}$. After step 2, $Y_{13}$ is already fixed. Thus we can use a simple calculation to get the value of $M_{4}$ :

$$
M_{4}=\left(X_{18} \boxminus X_{14}^{\lll 10}\right)^{\ggg 6} \boxminus \operatorname{IFX}\left(X_{17}, X_{16}, X_{15}^{\lll 10}\right) \boxminus Y_{13}
$$

- Step 4: The uncontrolled part of the merging. At this point, all freedom degrees have been used and the last equation on the internal state $X_{12}=Y_{12}$ will be fulfilled with a probability of $2^{-32}$.

Uncontrolled Probability. After the merging, steps 36-58 of the left branch and steps 29-58 of the right branch are still uncontrolled. Due to the difficulty of calculating the probability, we used experiments to evaluate these probabilities. Starting from a generated starting point, e.g. in Figure 4, we randomly choose values of message words $M_{5}, M_{9}, M_{0}, M_{6}, M_{13}, M_{1}, M_{7}$ and $M_{4}$. Then we compute forward to check if the differences are all canceled after the last injection 
of $M_{7}$. Note that if there are conditions on message words $M_{1}, M_{4}, M_{7}$ and $M_{13}$, they should be fulfilled probabilistically and included in the probability estimation, since the freedom degrees of these message words are used to match the initial internal states values. For the other free message words $M_{5}, M_{9}, M_{0}$ and $M_{6}$, we do not need to consider their conditions in the probability, because we can freely choose their values to fulfill these conditions.

We measured the probability of both branches separately. After applying the preparation trick, the uncontrolled probability of the left branch is $2^{-8.8}$. The uncontrolled probability of the right branch is $2^{-36.6}$. Moreover, during the merging phase, we could not control the value matching on the first IV word, and this adds another factor of $2^{-32}$.

In total, the uncontrolled probability is $2^{-32} \cdot 2^{-8.8} \cdot 2^{-36.6}=2^{-77.4}$. Since this probability is too low and already close to the birthday bound for RIPEMD-160, we are not able to afford the feed-forward tricks in steps 58-64.

Complexity Evaluation. First we calculate the complexity to generate the starting points. Since the uncontrolled probability is $2^{-77.4}$, we need to generate $2^{77.4}$ starting points. However, we do not need to restart the generation from the beginning. Indeed, every time we need a new starting point, we can randomize $M_{6}$ to get a new one. Once all possible choices of $M_{6}$ have been used, we can still use freedom degrees of $M_{0}, M_{9}$ and $M_{5}$ to generate all the required starting points. Though there are many constraints on these four message words, luckily the number of conditions on $M_{6}$ is only two bits (one on $X_{18}$ and one on $X_{17}$ ). We can randomly choose value for $X_{18}$ fulfilling the known conditions and check if the one-bit condition on $X_{17}$ is fulfilled. Thus, we can find a new starting point from a known one with a complexity of 4 step functions, which is equivalent to $4 /(42 * 2) \approx 2^{-4.4}$ calls of the 42 -step compression function of RIPEMD-160. For the other message words, we do not need to go into the details of the complexity, since the number of times we have to regenerate them is quite small and it is not the bottleneck of our attack complexity. From the reasoning above, we can conclude that the average complexity to generate a starting point is $2^{-4.4}$. The complexity of generating all the required starting points is then $2^{73}$.

Now, we need to consider the complexity of the merging phase. In order to evaluate this cost, we implemented the merging of the last four initial internal states. The table lookup in second phase is estimated using a RAM access (since the table will be very bog). In total, our implementation of the merging takes about 145 cycles. The OPENSSL implementation of RIPEMD-160 compression function on the same computer takes about 1040 cycles. Thus, 42 steps of the compression function takes about $1040 * 42 / 80=546$ cycles. Then we can say that our merging costs $145 / 546 \approx 2^{-1.9}$ calls of the 42 -step compression function.

Finally, we can calculate the complexity of the semi-free start collision attack on 42-step RIPEMD-160: $2^{73}+2^{77.4-1.9} \approx 2^{75.5}$. 


\subsection{First Step Semi-Free-Start Collision}

This section discusses about the merging phase of the two branches to get a semi-free-start collision attack on the the first 36 steps. The idea of the merging is similar to the merging for the 42-step attack and we describe it briefly.

We start with generating a starting point. After that, the path in the left branch has been satisfied until step 14, and the remaining uncontrolled probability amounts to $2^{-4.6}$. The path in the right branch has been fully satisfied. After that, there are free bits left in message words $M_{0}, M_{2}, M_{5}, M_{7}, M_{9}$ and $M_{14}$. Next, we show these free bits are enough to generate semi-free-start collisions, and thus we only need to generate a single starting point.

The procedure of merging is detailed as below.

1. Set random values to $M_{9}$ and the free bits of $M_{7}$, and then compute until $X_{2}$ in the left branch.

2. Set $M_{5}=M_{5} \boxplus 1$ (initialize $M_{5}$ as 0 ), and compute until $X_{-1}$ in the left branch. If $M_{5}$ becomes 0 again, goto Step 1 .

3. Compute the values of $M_{2}$ and $M_{0}$ that make $Y_{0}=X_{0}$ and $Y_{-1}=X_{-1}$.

4. Compute $X_{-2}$ and $Y_{-2}$, and check if $X_{-2}=Y_{-2}$ holds. In case of $X_{-2} \neq Y_{-2}$, goto Step 2.

5. Compute $X_{-3}$, and then compute the value of $M_{14}$ that makes $Y_{-3}=X_{-3}$. Check if the conditions on $M_{14}$ are satisfied. If the conditions are not satisfied, goto step 1.

6. Compute $X_{-4}$ and $Y_{-4}$, and check if $X_{-4}=Y_{-4}$ holds. In case of $X_{-4} \neq Y_{-4}$, goto Step 2.

Both $X_{-2}=Y_{-2}$ and $X_{-4}=Y_{-4}$ are satisfied with a probability $2^{-32}$, and four bit conditions are set on $M_{14}$. Thus we have to try $2^{68}$ random values of $M_{7}, M_{9}$, and $M_{5}$ to succeed in merging the two branches once. Recall that the uncontrolled probability is $2^{-4.6}$. So we need to merge the two branches $2^{4.6}$ times. Thus, the total complexity of the attack is $2^{68+4.6} \times 16 / 72 \approx 2^{70.4}$.

\section{Results}

We give in Table2 a comparison of our attacks to previous results on RIPEMD-160. Compared to the previous best semi-free-start collision attack on RIPEMD-160 (36 middle steps), we have increased the number of attackable steps by 6 and proposed a 36-step semi-free-start collision attack that starts from the first step.

\section{Conclusion}

In this article, we have proposed an improved cryptanalysis of the hash function RIPEMD-160, which is an ISO/IEC standard. We have found a 42-step semifree-start collision attack on RIPEMD-160 starting from the second step and a 36-step semi-free-start collision attack starting from the first step. Compared to previous results, we have two improvements. First the number of attacked steps 
Table 2. Summary of known and new preimage and collision attacks on RIPEMD-160 hash and compression function

\begin{tabular}{|c|c|c|c|c|c|c|}
\hline Function & Size & Target & Attack Type & \#Steps & Complexity & Ref. \\
\hline RIPEMD-160 & 160 & comp. function & preimage & 31 & $2^{148}$ & 14 \\
\hline RIPEMD-160 & 160 & hash function & preimage & 31 & $2^{155}$ & 14 \\
\hline RIPEMD -160 & 160 & comp. function & semi-free-start collision & 36 & low & [10] \\
\hline RIPEMD-160 & 160 & comp. function & semi-free-start collision & 42 & $2^{75.5}$ & new \\
\hline RIPEMD-160 & 160 & comp. function & semi-free-start collision & 36 & $2^{70.4}$ & new \\
\hline RIPEMD-160 & 160 & comp. function & non-randomness & 48 & low & 10 \\
\hline RIPEMD-160 & 160 & comp. function & non-randomness & 51 & $2^{158}$ & 16 \\
\hline
\end{tabular}

is increased from 36 to 42 , and secondly, for the same number of attacked steps, we propose an attack that starts from the first step. Moreover, our semi-freestart collision attacks give a positive answer to the open problem raised in [10], in which the authors were not able to find any non-linear differential path in the first step, due to the XOR function that makes the non-linear part search much harder.

Our 42-step semi-free-start attack is obtained from a 48-step differential path. Unfortunately, we couldn't add these extra 6 steps to our attack without reaching a complexity beyond the birthday bound (this extra part would be verified with probability $2^{-11.3}$ ). Future works might include improving the probabilistic part even further. If one can improve this part by a factor of about $2^{7}$, a 48 -step semifree-start collision attack would then be obtained directly with our proposed differential path. Another possible improvement would be that if one can find a better non-linear differential path in the first round, it might be possible to merge both branches at the same time to a given $I V$ and eventually obtain a hash function collision.

Acknowledgments. The authors would like to thank the anonymous referees for their helpful comments. Thomas Peyrin, Lei Wang and Shuang Wu are supported by the Singapore National Research Foundation Fellowship 2012 (NRFNRFF2012-06).

\section{References}

1. Bertoni, G., Daemen, J., Peeters, M., Van Assche, G.: The Keccak reference. Submission to NIST (Round 3) (January 2011), http://csrc.nist.gov/groups/ST/ hash/sha-3/Round3/submissions_rnd3.html

2. Bosselaers, A., Preneel, B. (eds.): RIPE 1992. LNCS, vol. 1007. Springer, Heidelberg (1995)

3. Damgård, I.: A Design Principle for Hash Functions. In: Brassard, G. (ed.) CRYPTO 1989. LNCS, vol. 435, pp. 416-427. Springer, Heidelberg (1990) 
4. De Cannière, C., Rechberger, C.: Finding SHA-1 Characteristics: General Results and Applications. In: Lai, X., Chen, K. (eds.) ASIACRYPT 2006. LNCS, vol. 4284, pp. 1-20. Springer, Heidelberg (2006)

5. Dobbertin, H.: RIPEMD with Two-Round Compress Function is Not CollisionFree. J. Cryptology 10(1), 51-70 (1997)

6. Dobbertin, H., Bosselaers, A., Preneel, B.: RIPEMD-160: A Strengthened Version of RIPEMD. In: Gollmann, D. (ed.) FSE 1996. LNCS, vol. 1039, pp. 71-82. Springer, Heidelberg (1996)

7. International Organization for Standardization: Information technology - Security techniques - Hash-functions - Part 3: Dedicated hash-functions. ISO/IEC 101183:2004 (2004)

8. Landelle, F., Peyrin, T.: Cryptanalysis of Full RIPEMD-128. In: Johansson, T., Nguyen, P.Q. (eds.) EUROCRYPT 2013. LNCS, vol. 7881, pp. 228-244. Springer, Heidelberg (2013)

9. Leurent, G.: Analysis of Differential Attacks in ARX Constructions. In: Wang, X., Sako, K. (eds.) ASIACRYPT 2012. LNCS, vol. 7658, pp. 226-243. Springer, Heidelberg (2012)

10. Mendel, F., Nad, T., Scherz, S., Schläffer, M.: Differential Attacks on Reduced RIPEMD-160. In: Gollmann, D., Freiling, F.C. (eds.) ISC 2012. LNCS, vol. 7483, pp. 23-38. Springer, Heidelberg (2012)

11. Mendel, F., Nad, T., Schläffer, M.: Finding SHA-2 Characteristics: Searching through a Minefield of Contradictions. In: Lee, D.H., Wang, X. (eds.) ASIACRYPT 2011. LNCS, vol. 7073, pp. 288-307. Springer, Heidelberg (2011)

12. Mendel, F., Nad, T., Schläffer, M.: Improving Local Collisions: New Attacks on Reduced SHA-256. In: Johansson, T., Nguyen, P.Q. (eds.) EUROCRYPT 2013. LNCS, vol. 7881, pp. 262-278. Springer, Heidelberg (2013)

13. Merkle, R.C.: One Way Hash Functions and DES. In: Brassard, G. (ed.) CRYPTO 1989. LNCS, vol. 435, pp. 428-446. Springer, Heidelberg (1990)

14. Ohtahara, C., Sasaki, Y., Shimoyama, T.: Preimage Attacks on Step-Reduced RIPEMD-128 and RIPEMD-160. In: Lai, X., Yung, M., Lin, D. (eds.) Inscrypt 2010. LNCS, vol. 6584, pp. 169-186. Springer, Heidelberg (2011)

15. Rivest, R.L.: The MD4 Message-Digest Algorithm. IETF Request for Comments (RFC) 1320 (1992), http://www.ietf.org/rfc/rfc1320.html

16. Sasaki, Y., Wang, L.: Distinguishers beyond Three Rounds of the RIPEMD-128/160 Compression Functions. In: Bao, F., Samarati, P., Zhou, J. (eds.) ACNS 2012. LNCS, vol. 7341, pp. 275-292. Springer, Heidelberg (2012)

17. Wang, X., Lai, X., Feng, D., Chen, H., Yu, X.: Cryptanalysis of the Hash Functions MD4 and RIPEMD. In: Cramer, R. (ed.) EUROCRYPT 2005. LNCS, vol. 3494, pp. 1-18. Springer, Heidelberg (2005)

18. Wang, X., Yin, Y.L., Yu, H.: Finding Collisions in the Full SHA-1. In: Shoup, V. (ed.) CRYPTO 2005. LNCS, vol. 3621, pp. 17-36. Springer, Heidelberg (2005)

19. Wang, X., Yu, H.: How to Break MD5 and Other Hash Functions. In: Cramer, R. (ed.) EUROCRYPT 2005. LNCS, vol. 3494, pp. 19-35. Springer, Heidelberg (2005)

20. Wang, X., Yu, H., Yin, Y.L.: Efficient Collision Search Attacks on SHA-0. In: Shoup, V. (ed.) CRYPTO 2005. LNCS, vol. 3621, pp. 1-16. Springer, Heidelberg (2005) 


\section{A The Differential Paths}

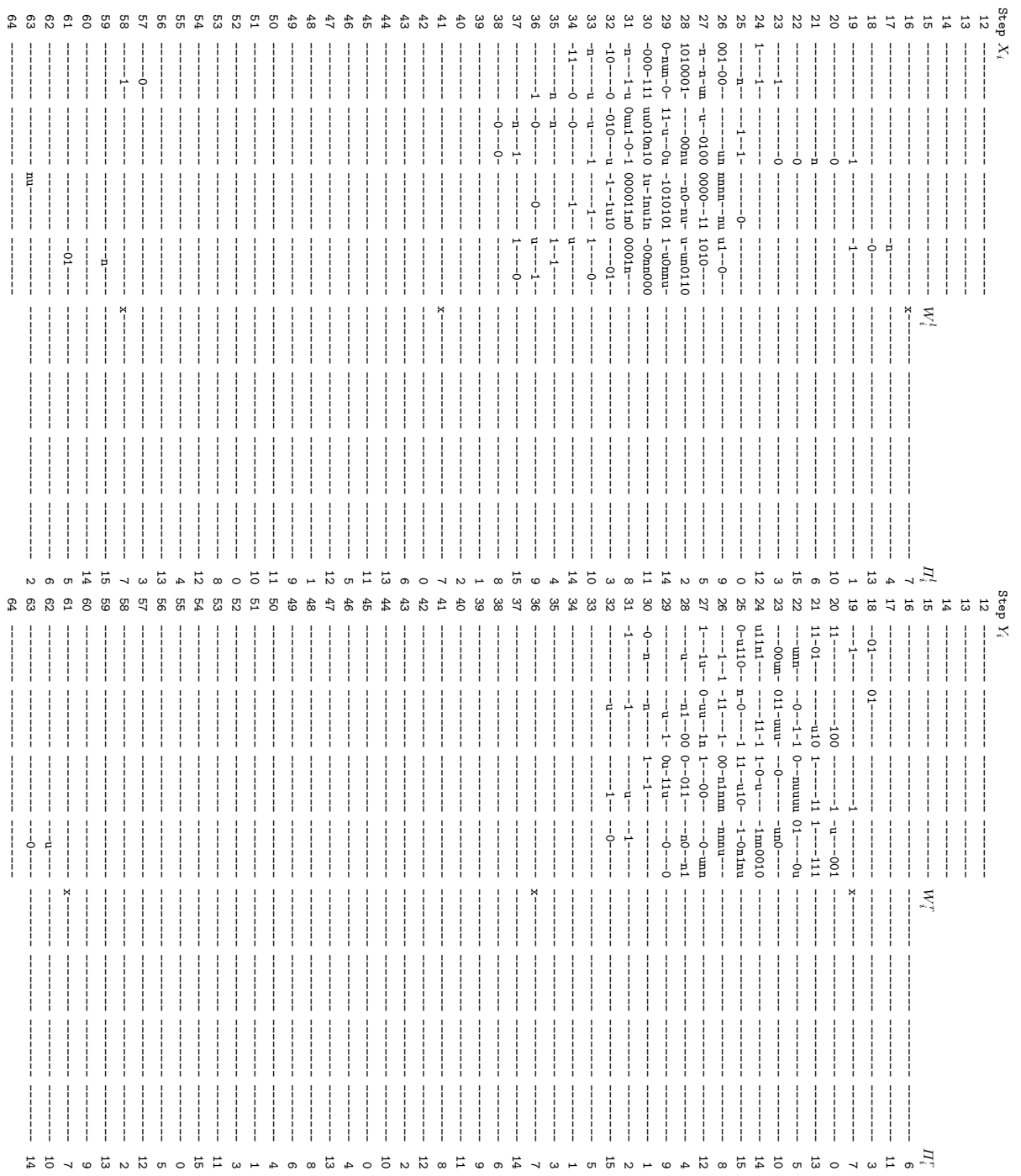

Fig. 5. The 48-step differential path 


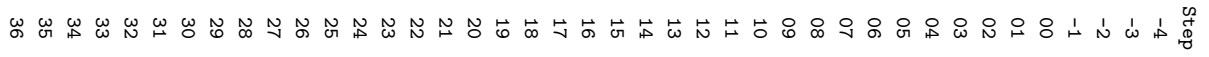

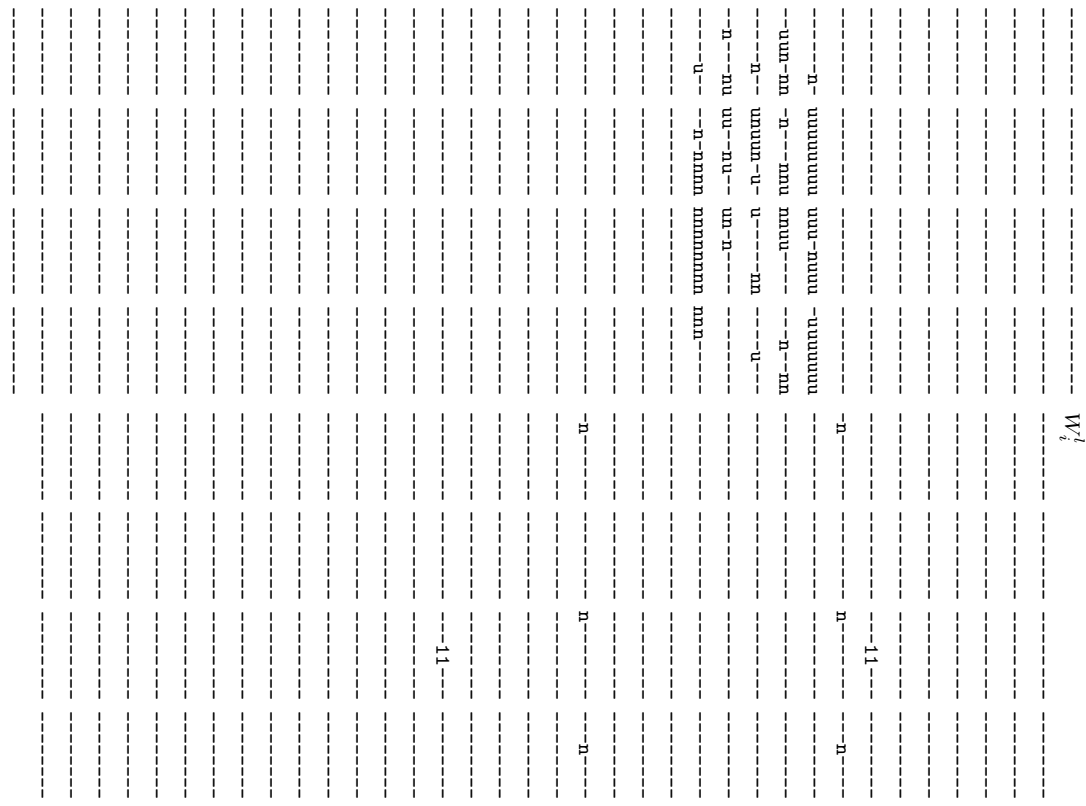

ه 台台 $\omega$ D

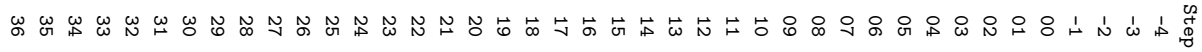

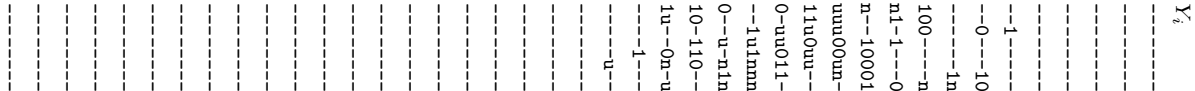

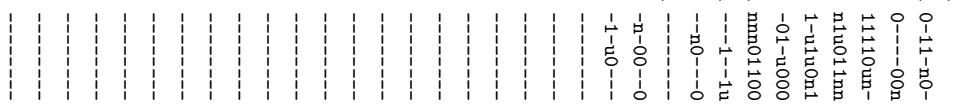

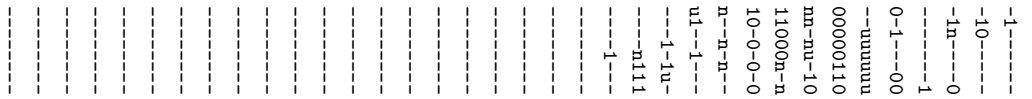

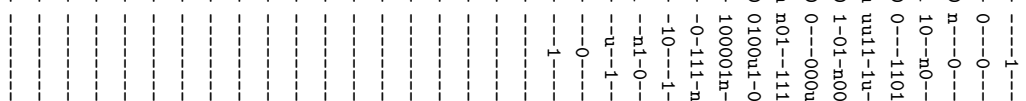

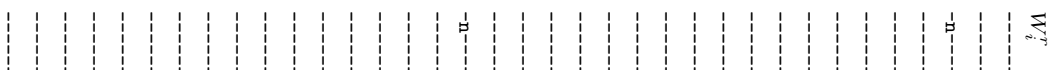

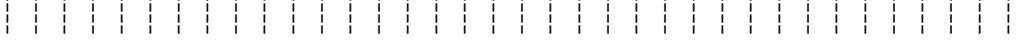

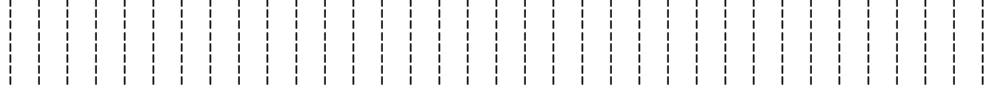

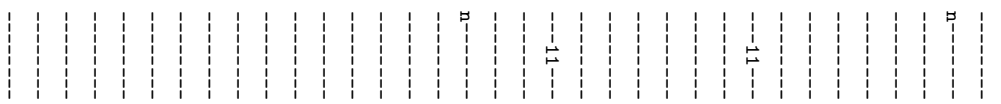

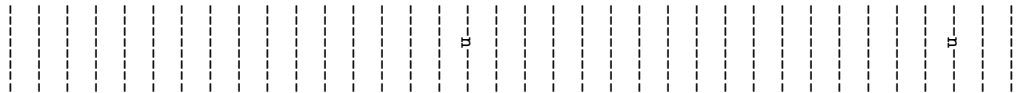

$\omega \mapsto$ G

Fig. 6. The 36-step differential path 